\title{
A systematic review and meta-analysis of randomized controlled trials: Efficacy and safety of larazotide acetate in celiac disease patients undergoing a gluten challenge
}

\author{
Harsha Moole ${ }^{1,2 *}$, Jaymon Patel ${ }^{1,2}$, Sowmya Dharmapuri ${ }^{3}$, Raghuveer Boddireddy ${ }^{4}$, Vishnu \\ Mool $^{\text {5 }}$, Prathyusha Yedama ${ }^{6}$, Naveen Bondalapati ${ }^{7}$, Achuta Uppu ${ }^{8,9}$, Charan Yerasi ${ }^{10}$ \\ ${ }^{1}$ Division of General Internal Medicine, University of Illinois College of Medicine at Peoria, Illinois, United States \\ ${ }^{2}$ Department of Internal Medicine, OSF St. Francis Medical Center, Peoria, Illinois, United States \\ ${ }^{3}$ Division of General Internal Medicine, Deccan Medical College, NTR University of Health Sciences, Andhra Pradesh, India \\ ${ }^{4}$ Division of General Internal Medicine, Pinnamaneni Siddhartha Medical College, NTR University of Medical Sciences, Andhra Pradesh, India \\ ${ }^{5}$ Division of General Internal Medicine, Mamatha Medical College, NTR University of Health Sciences, Andhra Pradesh, India \\ ${ }^{6}$ Division of General Internal Medicine, S.V.S Medical College, NTR University of Health Sciences, Andhra Pradesh, India \\ ${ }^{7}$ Division of Medicine, Barnes Jewish Christian Medical Group, Christian Hospital, St. Louis, Missouri, United States \\ ${ }^{8}$ Department of Medicine, Bronx Lebanon Hospital Center, Bronx, New York, United States \\ ${ }^{9}$ Department of Medicine, Albert Einstein College of Medicine, Bronx, New York, United States \\ ${ }^{10}$ Department of Medicine, Med Star Georgetown University Hospital / MedStar Washington Hospital Center, Washington, DC, DC, United States
}

Received: February 29, 2016; Accepted: May 01, 2016; Published: June 01 , 2016

*Corresponding author: Harsha Moole, Department of Internal Medicine, University of Illinois College of Medicine Peoria, 530 NE Glen Oak Ave, Peoria, Illinois, USA. 61637, Tel: (309) 655-2000, Email: harsha1778@yahoo.co.in

\begin{abstract}
Background: Celiac disease pathogenesis involves increased permeability of intestinal epithelial tight junctions due to immunogenic mechanisms. Larazotide acetate is a tight junction regulator peptide that was recently described.
\end{abstract}

Aims: To assess the efficacy and safety of larazotide acetate in celiac disease

Methods: Study Selection Criteria: Studies evaluating the efficacy and safety of larazotide acetate (LA group) in patients with celiac disease who were simultaneously challenged with gluten.

Data collection \& extraction: Articles were searched in Medline, Pubmed, and Ovid journals.

Statistical Method: Pooled proportions were calculated using fixed and random effects model.

Results: Initial search identified 89 reference articles, of which 19 were selected. Three studies $(\mathrm{N}=136)$ met the inclusion criteria. In the pooled proportion of patients, the change in urinary lactulose to mannitol fractional excretion ratio (LAMA) scores from baseline after the intervention in LA group and placebo group was $1.34(95 \% \mathrm{CI}=0.96$ to 1.71$)$ and $1.91(95 \% \mathrm{CI}=1.50$ to 2.32$)$ respectively. Change in Gastrointestinal Symptom Rating scale (GSRS) scores after intervention was $0.04(95 \% \mathrm{CI}=-0.32$ to 0.40$)$ and $0.46(95 \% \mathrm{CI}=0.09$ to 0.83 ) in LA and placebo group respectively. Change in Celiac disease GSRS (CeD-GSRS) scores after intervention was $-0.11(95 \% \mathrm{Cl}=-0.48$ to 0.26 ) and $0.35(95 \% \mathrm{CI}=-0.29$ to 0.99 in $\mathrm{LA}$ and placebo groups respectively. Odds ratio for overall adverse events (gastrointestinal symptoms, headache, urinary tract infection, fatigue) in LA group versus placebo group was $0.60(95 \% \mathrm{CI}=0.28$ to 1.28$)$.

Conclusions: Lower doses of Larazotide acetate seemed to control gastrointestinal symptom severity induced by the gluten challenge in celiac patients. It has a relatively safe side effect profile compared to placebo.

Keywords: Larazotide Acetate; Celiac Disease; Outcomes; Meta-Analysis And Systematic Review

\section{Introduction}

Celiac disease is a chronic autoimmune disorder in genetically susceptible individuals that results in intestinal, and often extraintestinal, manifestations in response to the ingestion of gluten, a mixture of proteins found in barley, rye, or wheat-products [1, 2 , and 3]. Nearly $100 \%$ of affected individuals have HLA-DQ2 or HLA-DQ8 positivity. The most common intestinal manifestations include symptoms of bloating, abdominal pain, and diarrhea [4, 5]. Extra-intestinal manifestations occur on a spectrum and may range from iron-deficiency anemia, infertility, and osteoporosis to malignancy including intestinal adenocarcinoma and nonHodgkin's lymphoma. These manifestations are likely result of chronic inflammation and stimulation of intestinal lymphocytes $[6,7]$. When compared with healthy controls, patients with 
celiac disease have increased intestinal permeability and altered tight junctions that contribute to pathogenesis $[8,9$, and 10]. Exposure of intestinal epithelial cells to gluten results in a cytoskeletal rearrangement that allows the entry of gluten peptides into the lamina propria via transcellular and paracellular transport [11,12,13, and 14]. Once in the lamina propria, tissue transglutaminase modifies the gluten peptides and bolsters their immunogenicity via engaging with MHC class II molecules [15]. The ensuing cytokine release then leads to a prolonged duration of tight-junction opening, resulting in increased permeability and greater influx of protein, thereby perpetuating the inflammatory response in a cyclic manner $[16,17]$.

Presently, subjects with celiac disease are managed via dietary-restriction to maintain a gluten-free diet (GFD). However, adherence to maintaining a GFD remains challenging as a result of the nearly ubiquitous nature of gluten and its presence in foods, often resulting in inadvertent exposure. Inadvertent exposure may result in up to two grams of gluten ingestion daily $[18,19]$. Additionally, even small, seemingly inconsequential quantities of gluten can result in symptoms and ongoing, chronic inflammation and failure of mucosal healing. Therefore, maintenance of a GFD alone is likely insufficient to manage a patient with celiac disease and the need for pharmacological intervention is needed.

Larazotideacetate, also known as AT-1001, is a first-inclass synthetic octapeptide that in vitro inhibits paracellular permeability by preventing the opening of tight junctions induced by various stimuli. These stimuli include cytokines, gluten peptides, and bacterial antigens $[20,21]$. The immunological activity of Larazotide acetate is limited in location to the luminal surface of the small intestine [22] and its mechanism of action involves the inhibition of cytoskeleton rearrangement, disassembly of tight junction and peak F-actin increment [23, $24,25]$. Larazotide acetate use resulted in sustained tight junction structure, decreased marker-protein transport across the intestinal epithelium, and a diminished macrophage count in the lamina propria in a transgenic mouse model of glutensensitive enteropathy [20]. Three randomized controlled trials evaluated the efficacy and safety of Larazotide acetate, and have shown mixed outcomes. This is the first meta-analysis to pool the evidence for Larazotide acetate in patients with celiac disease.

\section{Methods}

\section{Aims}

Primary outcome was to assess the efficacy of larazotide acetate in celiac disease patients. Surrogate marker for intestinal permeability, urinary lactulose to mannitol fractional excretion ratio (LAMA) was assessed. Gastrointestinal symptom rating scale (GSRS) and Celiac disease GSRS (CeD-GSRS) were monitored. Secondary outcome was to assess the overall safety profile of larazotide acetate.

\section{Study selection criteria:}

\section{Inclusion criteria:}

- Subjects needed confirmation of the presence of celiac disease via biopsy and positive antibody screen.
- Subjects needed to follow a gluten-free diet for at least 6 months prior to enrollment in the study, and needed to have anti-tissue transglutaminase titers of less than $10 \mathrm{EU}$ at study entry.

- Randomized controlled trials comparing the efficacy and safety of larazotide acetate in treatment group ( received Larazotide acetate $1 \mathrm{mg}$ orally, three times daily) (LA group) versus placebo group in patients with celiac disease who were simultaneously challenged with $2.4-$ 2.7 gm gluten ingestion per day during the trial.

- If women were enrolled in the studies, women must have been post-menopausal, surgically sterile, or must have had a negative pregnancy screening and agreed to acceptable forms of contraception.

- Studies with patient age 18 to 72 years were included in this study. Individual studies included 18-65 years of age in Leffler et al [26], 18-72 years of age in Kelly et al [27], and 18-59 years of age in Paterson et al [22].

Exclusion criteria: Studies without a control / placebo arm were excluded. Studies that were done in patients without a simultaneous gluten challenge were excluded. Among the individual studies: Patients were excluded from the trials if they had other chronic active gastrointestinal diseases, food intolerances or food allergies (other than to gluten), had a history of diabetes mellitus, used nicotine-containing products within 6 months of study entry, a history of HIV, hepatitis B, hepatitis C, were pregnant or breast-feeding, had a history of alcohol or drug abuse, had a history of major active psychiatric or neurological disease, were taking medications known to result in immunosuppression or alter intestinal functioning, permeability, or $\mathrm{pH}$, used were on antibiotics, laxatives, herbal supplements, probiotics, amphetamines, or pancreatic enzyme replacement therapy, or participated in a clinical trial within 30 days of randomization.

Data collection \& extraction: Articles were searched in Medline, PubMed, Ovid journals, EMABSE, Cumulative Index for Nursing \& Allied Health Literature, ACP journal club, DARE, International Pharmaceutical Abstracts, old Medline, Medline non indexed citations, OVID Health star, and Cochrane Central Register of Controlled Trials (CENTRAL). The search was performed for the years 1966 to January 2016. Abstracts were manually searched in the major gastroenterology, internal medicine journals for the past three years. Study authors for the abstracts included in this analysis were contacted when the required data for the outcome measures could not be determined from the publications. The search terms used were Larazotide acetate, AT-1001, randomized controlled trial, celiac disease, coelic disease, gluten-free, and gluten challenge. Two authors (HM and VM) independently searched and extracted the data into an abstraction form. Any differences were resolved by mutual agreement. The agreement between reviewers for the collected data was quantified using the Cohen's $\kappa$ [28]. 
Quality of Studies: Clinical trials designed with a control and treatment arms can be assessed for quality of the study. A number of criteria have been used to assess this quality of a study (e.g. randomization, selection bias of the arms in the study, concealment of allocation, and blinding of outcome) $[29,30]$.

Statistical methods: This meta-analysis was performed by calculating pooled proportions. First the individual study proportion of all the study variables was transformed into a quantity using Freeman-Tukey variant of the arcsine square root transformed proportion. The pooled proportion is calculated as the back-transform of the weighted mean of the transformed proportions, using inverse arcsine variance weights for the fixed effects model and DerSimonian-Laird weights for the random effects model [31, 32]. Forest plots were drawn to show the point estimates in each study in relation to the summary pooled estimate. The width of the point estimates in the Forest plots indicates the assigned weight to that study. The heterogeneity among studies was tested using Cochran's $Q$ test based upon inverse variance weights [33]. If $p$ value is $>0.10$, it rejects the null hypothesis that the studies are heterogeneous. The effect of publication and selection bias on the summary estimates was tested by both Harbord-Egger bias indicator [34] and BeggMazumdar bias indicator [35]. Also, funnel plots were constructed to evaluate potential publication bias [36, 37]. Microsoft Excel 2013 software and Revman were used to perform statistics for this meta-analysis.

Definitions: The primary efficacy outcome were measured via the urinary lactulose to mannitol ratio, questionnaires including the GSRS (38-40,42-44) and the Psychological General Well-Being Index (PGWBI) [41, 42, 45, 46] The lactulose to mannitol ratio served as a marker of intestinal permeability where mannitol absorption would be reduced as a result of mucosal injury and para-cellular absorption of lactulose would be increased. GSRS is a patient completed questionnaire that has 15 questions divided among five domains (reflux, constipation, diarrhea, indigestion and abdominal pain).Based on celiac disease symptoms, scores from three of these domains (indigestions, abdominal pain and diarrhea) were together referred as CeD-GSRS [26].

\section{Results}

Initial search identified 89 reference articles, in which 19 articles were selected and reviewed. Data was extracted from three studies [22, 26, and 27] that evaluated the efficacy and safety of Larazotide acetate in celiac disease patients, which met the inclusion criterion. All the studies are published as full text articles. Figure 1 shows the flow diagram of search results. All the pooled estimates given are estimates calculated by the fixed effect model. All the three studies included in this meta-analysis were randomized controlled trials (RCT).

The total number of patients included in this meta-analysis is 136 , with a female population (71\%). Median age of the patients was 46 years. Patients in LA group were treated with larazotide acetate $1 \mathrm{mg}$ orally, three times daily for a range of one to six weeks, with a median of two weeks. Table 1 shows the baseline characteristics of the studies. The $\mathrm{p}$ for chi-squared heterogeneity for all the pooled accuracy estimates was $>0.10$. The agreement between reviewers for the collected data gave a Cohen's $\kappa$ value of 1.0 .

\section{Efficacy of Larazotide acetate}

After the treatment interventions, in the pooled patient population, the change in LAMA scores from baseline after the intervention in LA group and placebo group was 1.34 (95\% CI $=0.96$ to 1.71$)$ and $1.91(95 \% \mathrm{CI}=1.50$ to 2.32$)$ respectively.

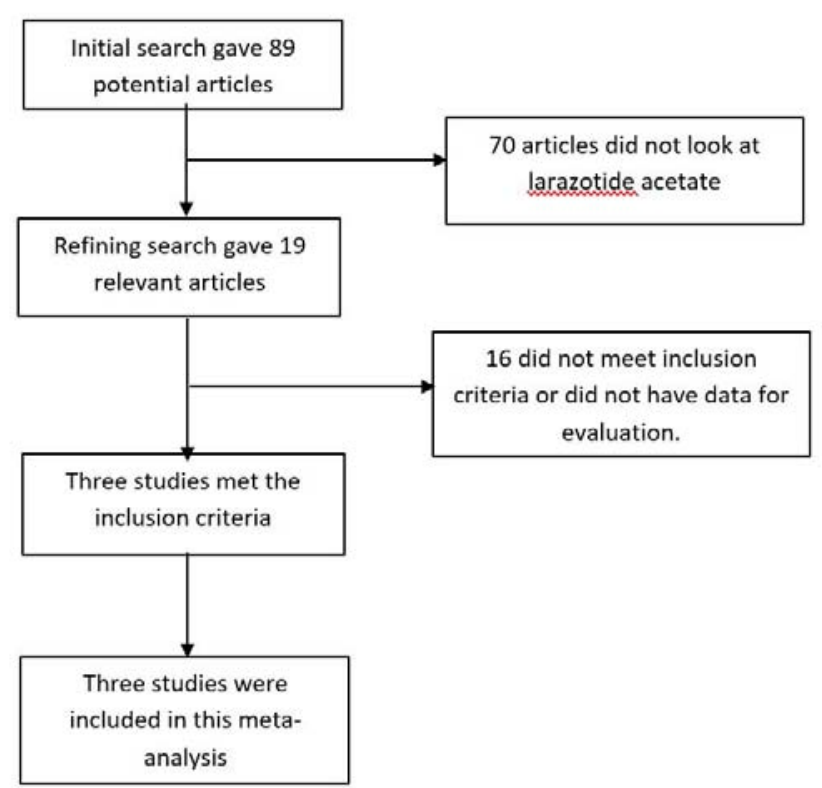

Figure 1: Flow diagram: Search results. 

challenge

Table 1: Basic characteristics of the included studies.

\begin{tabular}{|c|c|c|c|c|c|c|c|c|}
\hline No & Study & Type & Gluten challenge & Age in years-median & N & N-LA group & N-Placebo & Sex-Male \\
\hline 1 & Kelly et al, 2013 & RCT & Yes; $2.7 \mathrm{gm} /$ day & 50 & 89 & 45 & 44 & 24 \\
\hline 2 & Leffler et al, 2012 & RCT & Yes; $2.4 \mathrm{gm} /$ day & 46 & 26 & 12 & 14 & 11 \\
\hline 3 & Paterson et al, 2007 & RCT & Yes; $2.5 \mathrm{gm} /$ day & 36 & 21 & 14 & 7 & 4 \\
\hline
\end{tabular}

RCT - Randomized controlled trial; N - Total number of patients in the study; N-LA group - Number of patients in LA group; N-Placebo - Number of patients in placebo

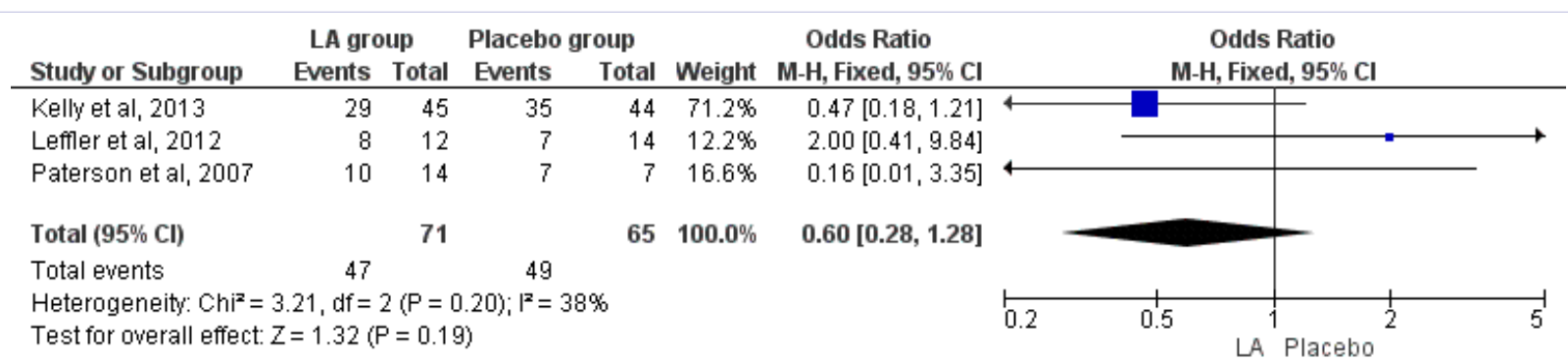

Figure 2: Forest plot - Individual study proportions and the pooled estimate for odds ratio of adverse events in LA group vs. placebo group (fixed effects).

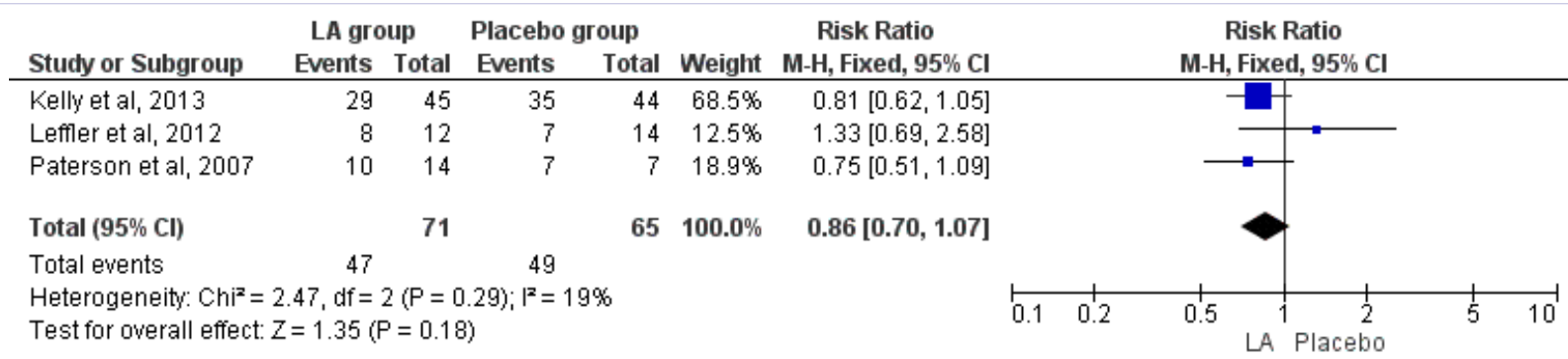

Figure 3: Forest plot - Individual study proportions and the pooled estimate for risk ratio of adverse events in LA group vs placebo group (fixed effects).

Heterogeneity was assessed using $\mathrm{I}^{2}$ (inconsistency) $=0 \%(95 \%$ $\mathrm{CI}=0 \%$ to $72.9 \%$ ).

Change in GSRS scores after intervention was $0.04(95 \% \mathrm{CI}=$ -0.32 to 0.40$)$ and $0.46(95 \% \mathrm{CI}=0.09$ to 0.83$)$ in $\mathrm{LA}$ and placebo group respectively. Change in CeD-GSRS scores after intervention was $-0.11(95 \% \mathrm{CI}=-0.48$ to 0.26$)$ and $0.35(95 \% \mathrm{CI}=-0.29$ to 0.99 in LA and placebo groups respectively. Heterogeneity was assessed using $\mathrm{I}^{2}$ (inconsistency) $=0 \%(95 \% \mathrm{CI}=0 \%$ to $72.9 \%)$. Bias indicator was Horbold-Egger: bias $=-0.13(92.5 \% \mathrm{CI}=-27.07$ to 26.79) $\mathrm{P}=0.97$.

\section{Adverse events with Larazotide acetate}

In the pooled patient population, the odds ratio for overall adverse events (gastrointestinal symptoms, headache, urinary tract infection, fatigue) in LA group versus placebo group was $0.60(95 \% \mathrm{CI}=0.28$ to 1.28$)$. There were no fatal adverse events in both groups. Due to the limited data available from individual studies, we were not able to pool the results for individual adverse events separately, cost benefit analysis and hospitalizations. Figure 2 and 3 are forest plot representing the odds ratio and risk ratio for overall adverse events in LAgroup vs placebo group. Figure 4 and 5 are funnel plots assessing the publication bias for same variables respectively. Figures 2 and 3 have information regarding heterogeneity of studies. Bias indicator was HorboldEgger: bias $=-0.13(92.5 \% \mathrm{CI}=-27.07$ to 26.79$) \mathrm{P}=0.97$.

\section{Discussion}

In each of the studies assessed, Larozotide acetate improved gastrointestinal symptoms in patients with Celiac disease. Kelly et al [27] conducted a randomized, double-blind, placebocontrolled, exploratory study across 20 sites in the United States and Canada that lasted for six weeks, involving 184 subjects aged 18-72 with celiac disease diagnosed via duodenal or jejunal biopsy at least 6 months prior to study entry. All subjects in the study were Caucasian with the exception of 1 individual, who was Hispanic. Subjects were randomized to receive aplacebo, $1 \mathrm{mg}$, $4 \mathrm{mg}$, or $8 \mathrm{mg}$ Larazotide acetate capsule three times daily 15 minutes before meals for a 42 day treatment period after a 7 day run-in period where all patients received placebo drug and placebo gluten capsules. They also received $900 \mathrm{mg}$ of gluten 


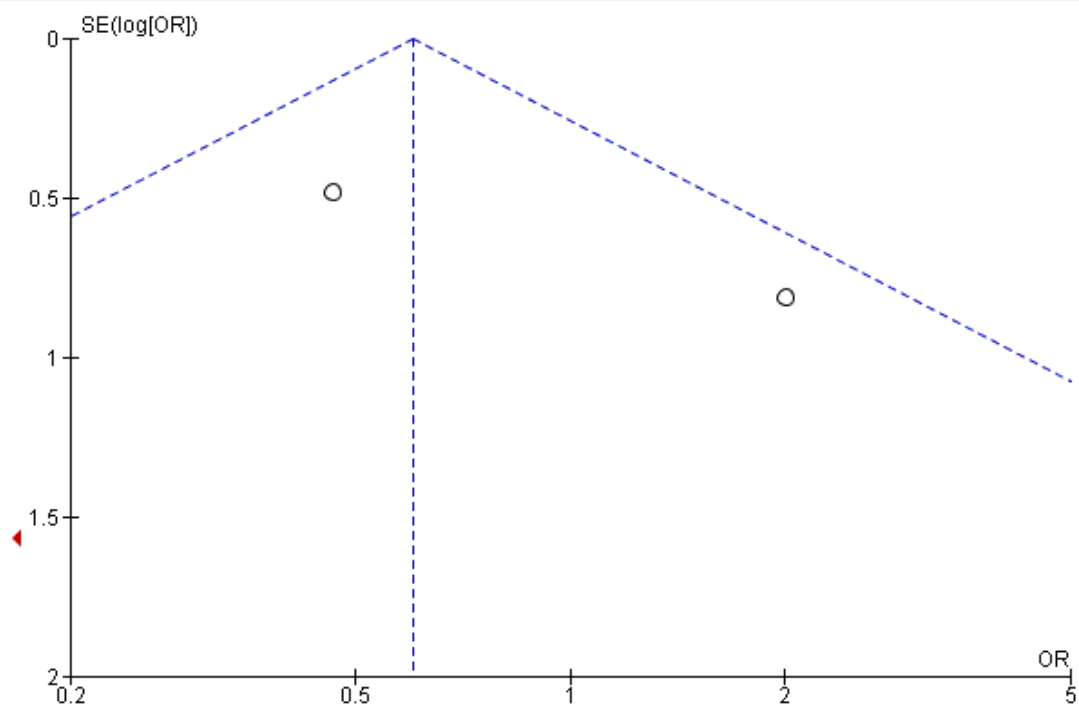

Figure 4: Funnel plot assessing for publication bias (odds ratio of adverse events in LA group vs placebo group).

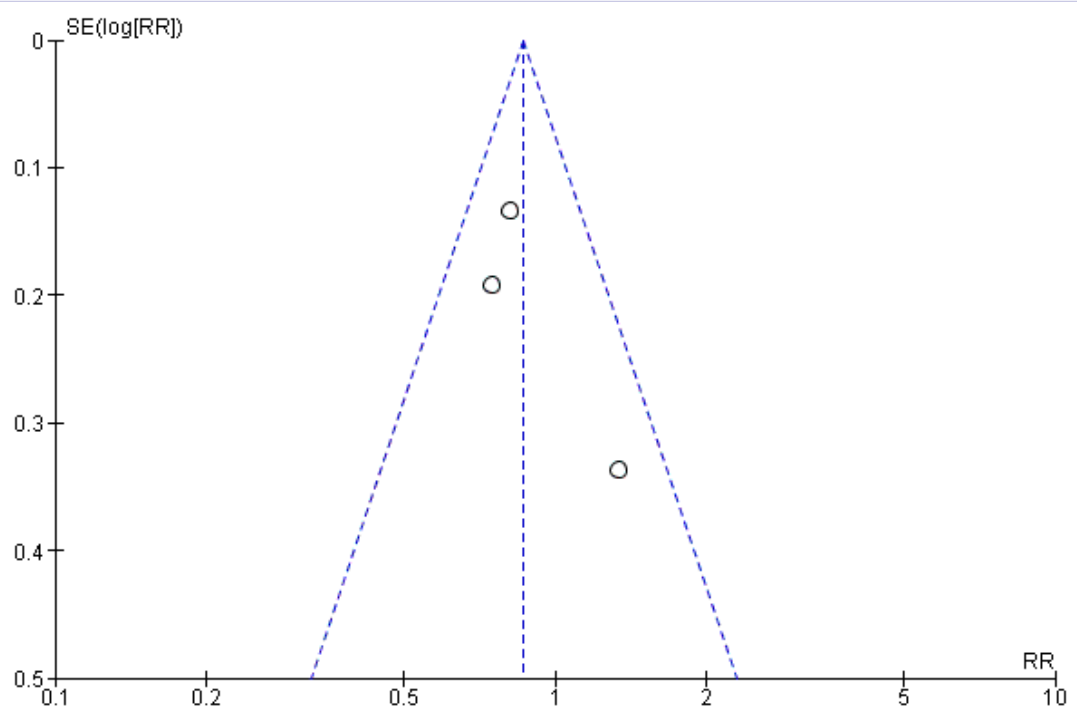

Figure 5: Funnel plot assessing for publication bias (risk ratio for adverse events in LA group vs placebo group).

three times daily with meals to simulate the effect of inadvertent gluten ingestion. The study found that Larazotide acetate led to a reduction of symptoms and gluten-induced immune reactivity in patients with celiac disease that underwent a gluten challenge. The primary endpoint, the lactulose to mannitol ratio at the last double-blind treatment period visit, was found to trend lower in the $1 \mathrm{mg}$ Larazotide acetate group than in the placebo group (approximately 1.7 vs 2.4 ). In the placebo group, the GSRS scores increased during the first 3 weeks of the gluten challenge, whereas the GSRS scores remained near baseline in the $1 \mathrm{mg}$ Larazotide acetate group. The GSRS revealed improvements in the indigestion, abdominal pain and diarrhea subgroups in the $1 \mathrm{mg}$ treatment group versus the placebo group with the most significant change being in the diarrhea sub-domain. The PGWBI scores were also found to be lower in the placebo group during the last 2 weeks of the gluten challenge, in comparison to the treatment groups whose scores remained near or above baseline. Other measures included measurement of anti-tissue transglutaminase IgA antibody levels that remained below $10 \mathrm{EU}$ in the $1 \mathrm{mg}$ and $4 \mathrm{mg}$ treatment groups, whereas $30 \%$ of subjects in the placebo group seroconverted. Larazotide acetate was well tolerated in the study with the most common adverse events involving gastrointestinal symptoms which were similar between the treatment and placebo groups, except for abdominal pain in the $8 \mathrm{mg}$ treatment group. Analysis via EKG, urinalysis, hematology and serum chemistry showed no clinically significant 

challenge

changes during the study, and antibodies to Larazotide acetate were not detected indicating lack of any significant systemic absorption.

Another study to assess the effect of Larazotide acetate in patients with celiac disease was conducted by Leffler et al [26]. The study was a prospective, double-blind, multicenter, placebocontrolled, randomized trial across ten clinical sites across the United States for a study period of 14 days. The study consisted of 86 patients aged 18 to 65 , of which, 53\% were women and all were Caucasian except one patient. In the study, patients were randomly assigned to one of seven treatment groups. Four groups received a gluten challenge with either doses of $0.25,1,4$, or $8 \mathrm{mg}$ of Larazotide acetate three times daily while the other three groups were part of a safety-control arm. Of the three safety control arm groups, one group received 8mg of Larazotideacetate without a gluten challenge (placebo gluten), the second group received placebo medication and placebo gluten, and the third group received placebo medication and a gluten challenge. Medications were ingested in capsule form three times daily 15 minutes prior to meal intake. For the gluten challenge, patients ingested two capsules of $400 \mathrm{mg}$ of gluten three times daily with meals, for a total of $2.4 \mathrm{~g}$ of gluten consumption daily. Placebo gluten consisted of $100 \%$ cornstarch and was also ingested three times daily with meals in the placebogluten groups. There were five clinic visits used for assessment at screening, days $0,7,14$, and 21 . The primary outcome measure was the change in lactulose to mannitol ratios from day 0 to day 14. Of the patients who received the gluten challenge, statistically significant differences were absent in the lactulose to mannitol ratios between the Larazotide acetate groups and the placebo group. Patients who received Larazotide acetate and gluten fared better than those who received gluten without Larazotide acetate. Statistically significant symptom severity decreases were found in the gluten-free control group and those who received Larazotide acetate with gluten when compared with those inwho received gluten without Larazotide acetate. During the gluten-challenge period (days 0-14), the GSRS and CeD-GSRS indicated increased gastrointestinal symptoms which subsequently decreased after completion of the gluten-challenge (days 14-21). Therefore, symptom severity was found to be increased in response to gluten but protection from exposure was provided by Larazotide acetate according to the questionnaires completed. Statistically significant protection was found in the Larazotide acetate groups with respect to severity of abdominal pain, indigestion, and reflux, and near-statistical significance for diarrhea. Interestingly, there was no statistically significant difference with respect to changes in the PGWBI between the Larazotide acetate groups and the gluten-challenge control group. Anti-tissue transglutaminase antibodies were assessed at screening and at the conclusion of the study on day 21 and were found to have no significant differences. Larazotide acetate was found to be well tolerated in the study with headache being the most common adverse event. No serious adverse events were reported and assessments periodically via serum chemistries, hematology, vital sign assessment, EKG and physical exam showed no significant changes from baseline. Five patients that received Larazotide acetate developed urinary tract infections in the course of the study, one of which was attempted for culture that eventually resulted negative. Plasma levels of Larazotide acetate were obtained on days 0,7 , and 14 and were below the detectable level of $0.5 \mathrm{ng} / \mathrm{ml}$, again indicating that systemic absorption of Larazotide acetate was absent.

Paterson et al [22] conducted an inpatient, randomized, double-blind, placebo-controlled, single center exploratory study also to assess the safety and tolerability of $12 \mathrm{mg}$ doses of Larazotide acetate. Twenty one subjects, age 18-59, were enrolled and randomized in a 2:1 manner for drug: Placebo for a seven day assessment period. Treatment days were on days 1,2 , and 3 but gluten was only administered on day 2 . On day 2 , all subjects received a $2.5 \mathrm{~g}$ oral gluten challenge in a blinded fashion. End points were evaluated on study days 1, 2, 3, and 7 . Subjects received either $12 \mathrm{mg}$ of Larazotide acetate via entericcoated capsule or a matching placebo once daily for 3 consecutive days and were admitted to the clinical research unit on day 1 . On day 1 , subjects received Larazotide acetate or a placebo, followed by sham-gluten 30 minutes later. Thirty minutes later, patients consumed the solution of sucrose, lactulose, and mannitol for assessment of intestinal permeability and then underwent an eight hour urine collection for assessment. Day 2 followed the regimen of day 1 , but all subjects received gluten instead of sham-gluten. The regimen for day 3 was identical to that of day 1. Following evaluation on day 3 , patients were discharged and followed up on day 7 for follow-up assessments. The primary outcome found a $70 \%$ increase in the lactulose-to-mannitol ratio from day 2 to day 1 in the placebo group and no increase in the treatment group indicating the protective effects of Larazotide acetate. The findings in the difference in intestinal permeability between the 2 groups nearly achieved statistical significance on day 2. Secondary outcomes of the study, fractional excretions of lactulose and mannitol, were similar but weaker. Cytokine release (interferon gamma) was used as a marker of immune activity, and was found to trend lower in the treatment group in comparison to the placebo group but did not reach statistical significance. Subjects also maintained a GI symptom diary during their study for documentation of symptoms. Both groups reported increased GI urgency on day 2, but only the placebo group reported ongoing increased severity of symptoms on day 3.An increased number of loose, instead of firm stools, was also reported in the placebo group but failed to reach statistical significance. As with the previous two studies discussed, all plasma concentrations of Larazotide acetate were below quantifiable levels (less than $0.05 \mathrm{ng} / \mathrm{ml}$ ). The majority of the adverse events were involving gastrointestinal symptoms and the most commonly reported event was diarrhea. In fact, every celiac disease related symptoms was found to be greater in the placebo group versus the Larazotide group except vomiting. For non-gastrointestinal adverse events, the most commonly reported event was headache. For each of the three studies discussed, specific numerical data regarding the lactulose to mannitol ratios were not consistently provided, but rather, the trend was discussed along with their respective graphical representations. 
When patients with celiac disease who were on a gluten free diet were challenged with dietary gluten, larazotide acetate seemed to control symptoms better than a placebo as evidenced by relatively smaller changes in LAMA scores from baseline compared to placebo group (1.34 vs 1.91). The Patients 'qualities of life also improved in the LA group as evidenced by relatively minimal increases on GSRS compared to the placebo group 0.04 vs 0.46 ); reduction in CeD-GSRS scores compared to placebo group (-0.11 vs 0.35$)$. There were no fatal adverse events in the LA group or the placebo group. The side effect profile was comparable in both groups (odds ratio 0.60 with $95 \% \mathrm{CI}=0.28$ to 1.28$)$.

Strengths of this meta-analysis include the high quality methodology of statistical analysis, and high quality methodology used in individual studies. All the studies included in this meta-analysis were double-blinded, randomized, and placebocontrolled. The studies by Kelly et al [27] and Leffler et al [26] were multi-center, outpatient studies whereas the study conducted by Paterson et al [22] was a single-center, inpatient study. Each of the studies involved well matched subjects with essential inclusion and exclusion criteria.

Limitations of this meta-analysis are: Due to the nature of data available from individual studies, we were not able to pool the evidence for individual adverse events separately, cost benefit analysis and hospitalizations. The number of studies included in this meta-analysis was small; three studies with relatively few test subjects. The follow up period used in the individual studies was not uniform. There is a need for large multi-centered prospective trials to further validate the current evidence about larazotide acetate.

Studies with statistically significant positive results tend to be published and cited. Additionally, smaller studies may show larger treatment effects compared to larger studies. This publication and selection bias may affect the summary estimates. The bias can be estimated using Egger bias indicators and the construction of funnel plots, whose shape can be affected by bias. In the present meta-analysis and systematic review, bias calculations both Egger [34] and Begg-Mazumdar [35] bias indicators showed no statistically significant bias. Furthermore, analysis using funnel plots showed no significant publication bias among the studies included in the present analysis.

\section{Conclusions}

In conclusion, Larazotide acetate led to a reduction of gastrointestinal symptoms and improvement in gluten tolerance in the celiac disease patients. The medication was well tolerated, had relatively few and minor adverse effects, and remained localized with respect to absorption within the small bowelas there were no detectable plasma levels found. Although larger studies will likely be needed to further assess the safety and efficacy of Larazotide acetate, it appears at this time to be a potentially viable and effective treatment option.

\section{References}

1. Green, Peter HR. "The many faces of celiac disease: clinical presentation of celiac disease in the adult population." Gastroenterology. 2005;128(4 Suppl 1):S74-S78.

2. Murray JA, Van Dyke C, Plevak MF, Dierkhising RA, Zinsmeister A., Melton LJ 3rd. Trends in the identification and clinical features of celiac disease in a North American community, 1950-2001. Clin Gastroenterol Hepatol. 2003;1(1):19-27.

3. Farrell, Richard J, Ciarán P, Kelly. “Celiac sprue.” New England Journal of Medicine 346.3; 2002: 180-188

4. Rubio-Tapia, Alberto, and Joseph A. Murray. "Celiac disease." Curr opin in gastroenterol. 2010;26(2): 116-122. doi: 10.1097/ MOG.0b013e3283365263

5. Ludvigsson JF, PH Green. Clinical management of coeliac disease. J Intern Med. 2011;269(6):560-571. doi: 10.1111/j.13652796.2011.02379.x.

6. Green PH, Fleischauer AT, Bhagat G, Goyal R, Jabri B and Neugut AI. Risk of malignancy in patients with celiac disease. The American journal of medicine. 2003;115(3):191-195.

7. Potter DD, Murray JA, Donohue J H, Burgart LJ, Nagorney DM, van Heerden JA, et al. The Role of Defective Mismatch Repair in Small Bowel Adenocarcinoma in Celiac Disease. Cancer research. 2004;64(19): 7073-7077.

8. E Smecuol, JC Bai, H Vazquez, Z Kogan, A Cabanne, S Nivelon, et al. Gastrointestinal permeability in celiac disease. Gastroenterology. 1997;112(4):1129-36.

9. Schulzke JD, Bentzel CJ, Schulzke I, Riecken EO, Fromm M. Epithelial tight junction structure in the jejunum of children with acute and treated celiac sprue. Pediatric research. 1998;43(4): 435-441.

10.Vogelsang $H$, Schwarzenhofer $M$, Steiner B, Wyatt J and Oberhuber G. In vivo and in vitro permeability in coeliac disease. Alimentary pharmacology \& therapeutics. 2001;15(9):1417-1425. doi: 10.1046/j.1365-2036.2001.01034.x.

11. Ciclitira PJ, Johnson MW, Dewar DH and Ellis HJ. The pathogenesis of coeliac disease. Molecular aspects of medicine. 2005;26(6), 421-458. doi: 10.1016/j.mam.2005.05.001

12. Jarvinen KM, Meddings J, Noone S, Sampson HA, Arrieta MC, and Nowak-Wegrzyn, A. Increased intestinal permeability in asymptomatic children with food allergy. Journal of Allergy and Clinical Immunology. 2006;117(2):S300. doiI: 10.1016/j.jaci.2005.12.1186

13. Clayburgh, Daniel R, Le Shen, and Jerrold R. Turner. "A porous defense: the leaky epithelial barrier in intestinal disease." Laboratory investigation. 84.3 (2004):282-291.

14.Sander, Guy R, Adrian G. Cummins, and Barry C. Powell. Rapid disruption of intestinal barrier function by gliadin involves altered expression of apical junctional proteins. FEBS Lett. 2005;579.21:48514855.

15. Jabri, Bana, Donald D. Kasarda, and Peter HR Green. "Innate and adaptive immunity: the yin and yang of celiac disease." Immunol Rev.2006;206(1):219-231.

16. Bruewer, M, Luegering, A, Kucharzik T, Parkos CA, Madara JL, Hopkins AM, et al. Proinflammatory Cytokines Disrupt Epithelial Barrier Function by Apoptosis-Independent Mechanisms. The 
Journal of Immunology. 2003;171(11):6164-6172. doi: 10.4049/ jimmunol.171.11.6164

17.Turner, Jerrold R. Molecular basis of epithelial barrier regulation: from basic mechanisms to clinical application. Am J Pathol. 2006;169(6):1901-1909.

18. Leffler DA, Edwards-George J, Dennis M, Schuppan D, Cook F, Franko D $\mathrm{L}$, et al. Factors that influence adherence to a gluten-free diet in adults with celiac disease. Digestive diseases and sciences. 2008;53(6):15731581. doi: $10.1007 / \mathrm{s} 10620-007-0055-3$

19. Anna Giberta, Montserrat Espadalera, Miguel Angel Canelab, Anna Sa'ncheza, Cristina Vaque' a and Magda Rafecas. Consumption of gluten-free products: should the threshold value for trace amounts of gluten be at 20,100 or $200 \mathrm{ppm}$ ?. European journal of gastroenterology \& hepatology. 2006;18(11): 1187-1195.

20.Gopalakrishnan S, Durai M, Kitchens K, Tamiz AP, Somerville R, Ginski $\mathrm{M}$, et al. Larazotide acetate regulates epithelial tight junctions in vitro and in vivo. Peptides. 2012; 35(1):86-94. doi: 10.1016/j. peptides.2012.02.015.

21. Gopalakrishnan S, Tripathi A, Tamiz AP, Alkan SS, Pandey NB Larazotide acetate promotes tight junction assembly in epithelial cells. Peptides. 2012; 35(1):95-101. doi: 10.1016/j.peptides.2012.02.016

22. Paterson BM, Lammers KM, Arrieta MC, Fasano A, Meddings JB. The safety, tolerance, pharmacokinetic and pharmacodynamic effects of single doses of AT-1001 in coeliac disease subjects: a proof of concept study. Aliment Pharmacol Ther. 2007;26(5):757-766.

23. Clemente MG, De Virgiliis S, Kang JS, Macatagney R, Musu MP, Di Pierro, et al. Early effects of gliadin on enterocyte intracellular signalling involved in intestinal barrier function. Gut. 2003;52(2):218-223.

24. Drago S, El Asmar R, Di Pierro M, Grazia Clemente M, Tripathi A, Sapone A, et al. Gliadin, zonulin and gut permeability: Effects on celiac and non-celiac intestinal mucosa and intestinal cell lines. Scand J Gastroenterol. 2006;41(4):408-419.

25. Wang, W, Uzzau S, Goldblum SE, Fasano A. Human zonulin, a potential modulator of intestinal tight junctions. J Cell Sci. 2000;113 Pt 24:44354440 .

26. Leffler DA, Kelly CP, Abdallah HZ, Colatrella AM, Harris LA, Leon $\mathrm{F}$, et al. A randomized, double-blind study of larazotide acetate to prevent the activation of celiac disease during gluten challenge. Am J Gastroenterol. 2012;107(10):1554-1562.

27. Kelly CP, Green PH, Murray JA, Dimarino A, Colatrella A, Leffler DA, et al. Larazotide acetate in patients with coeliac disease undergoing a gluten challenge: a randomised placebo-controlled study. Aliment Pharmacol Ther. 2013;37(2):252-262.

28. Brennan P, Silman A. Statistical methods for assessing observer variability in clinical measures. BMJ. 1992;304:1491-1494.

29. Jadad AR, Moore RA, Carroll D, Jenkinson C, Reynolds DJ, Gavaghan DJ et al. Assessing the quality of reports of randomized clinical trials: Is blinding necessary? Controlled Clin Trials. 1996;17:1-12.

30. Stroup DF, Berlin JA, Morton SC, Olkin I, Williamson GD, Rennie D, et al. Meta-analysis of observational studies in epidemiology: a proposal for reporting. Meta-analysis of observational studies in Epidemiology (MOOSE) group. JAMA 2000;283(15):2008-2012.
31. Stuart A, Ord JK. Kendall's Advanced Theory of Statistics (6th edition). London: Edward Arnold; 1994.

32. DerSimonian R, Laird N. Meta-analysis in Clinical Trials. Controlled Clinical Trials. 1986;7(3):177-188.

33. Deeks JJ. Systematic reviews of evaluations of diagnostic and screening tests. In Egger M, Smith GD, Altman DG (eds). Systematic Reviews in Health Care. Meta-analysis in context. London: BMJ Books; 2001.

34. Harbord RM, Egger M, Sterne JAC. A modified test for small-study effects in meta-analyses of controlled trials with binary endpoints. Stat Med. 2005;25(20):3443-3457.

35. Begg CB, Mazumdar M. Operating characteristics of a rank correlation test for publication bias. Biometrics. 1994;50(4):1088-10101.

36. Sterne JAC, Egger M, Davey-Smith G. Investigating and dealing with publication and other biases in meta-analysis. BMJ. 2001;323(7304):101-105.

37. Sterne JAC, Egger M. Funnel plots for detecting bias in meta-analysis: guidelines on choice of axis. J Clin Epidemiol. 2001;54(10):1046-1055.

38. Dimenäs E, Glise H, Hallerbäck B, Hernqvist H, Svedlund J, Wiklund I. Well-being and gastrointestinal symptoms among patients referred to endoscopy owing to suspected duodenal ulcer. Scand J Gastroenterol 1995;30(11):1046-1052 .

39. Dimenas E, Carlsson G, Glise H, Israelsson B, Wiklund I. Relevance of norm values as part of the documentation of quality of life instruments for use in upper gastrointestinal disease. Scand J Gastroenterol Suppl 1996;221:8-13

40. Revicki DA, Wood M, Wiklund I, Crawley J. Reliability and validity of the Gastrointestinal symptom rating scale in patients with gastroesophageal ref ux disease. Qual Life Res 1998;7(1):75-83.

41. Dupuy HJ. The psychological general well-being (PGWB) index. In: Wenger NK, Mattson ME, Furberg C, et al., (eds). Assessment of Quality of Life in Clinical Trials of Cardiovascular Therapies. Le Jacq Publishing: New York, 1984 pp.170-83.

42. Mustalahti K, Lohiniemi S, Collin P, et al. Gluten-free diet and quality of life in patients with screen-detected celiac disease. Eff Clin Pract 2002;5:105-113.

43. Lohiniemi S, Mäki M, Kaukinen K, Laippala P, Collin P.Gastrointestinal symptoms rating scale in coeliac disease patients on wheat starchbased gluten-free diets. Scand J Gastroenterol Suppl 2000;35(9):947949.

44. Hallert C, Granno C, Grant C, S. Hultén, G. Midhagen, M. Ström, et al. Quality of life of adult coeliac patients treated for 10 years. Scand J Gastroenterol Suppl 1998;33(9):933-938. DOI:10.1080/003655298750026949.

45. Ukkola A, Mäki M, Kurppa K, Collin P, Huhtala H, Kekkonen Let al. Diet improves perception of health and well-being in symptomatic but not asymptomatic, patients with celiac disease. Clin Gastroenterol Hepatol. 2011;9(2):118-123. doi: 10.1016/j.cgh.2010.10.011.

46. Hallert C, Svensson M, Tholstrup J, Hultberg B. Clinical trial: B vitamins improve health in patients with coeliac disease living on a gluten-free diet. Aliment Pharmacol Ther. 2009;29(8):811-6. doi: 10.1111/j.1365-2036.2009.03945.x. 